\section{Free Radicals in Some Natural Resins}

CARL LAGERCRANTZ and M A R G A RE T A Y HLA N D

\section{Department of Medical Physics, University of} Göteborg, Sweden

$\mathrm{I}^{\mathrm{n}}$ this laboratory we have found that Lome resins derived from pine trees contain varying amounts of free radicals. Thus, the fossile resin amber, fresh oleoresin from Pinus silvestris, colophony (gum rosin) and canada balsam exhibited an electron spin resonance (ESR) absorption corresponding to a concentration of $10^{14}$ $10^{16}$ spins per gram substance. The ESRspectra consisted of a single line of a width between 10 and 15 gauss. The shape of the absorption curve was slightly asymmetrical for some of the specimens investigated. The $g$-value was close to that of diphenylpicrylhydrazyl.

When the samples were kept at an elevated temperature, the ESR-signal was reduced or completely abolished. Thus, the signal from a sample of amber was reduced by a factor of five when kept at $170^{\circ} \mathrm{C}$ for $10 \mathrm{~min}$. When a specimen of colophony had been melted in the ESRsample tube by heating to $120^{\circ} \mathrm{C}$ for some minutes and subsequently was allowed to solidify by cooling to room temperature, it was found that the ESR-signal had almost completely disappeared.

Grinding of such a previously melted and solidified specimen to a finely divided powder restored the ESR-signal. The reappearence and the subsequent increase of the ESR-signal was easily observed when the spectrum was recorded immediately after the specimen had been ground.

When a sample of colophony which exhibited a strong ESR-signal in the powdered form, was dissolved in xylene in the ESR-sample tube, no signal could be detected. An ESR-signal was still present, however, after the specimen had been dissolved in alkaline ethanol.

Irradiation by light from a xenon lamp of a melted and solidified specimen of faintly yellow colophony also restored the signal. Fig. 1 shows the relative radical concentration as a function of time when the sample was irradiated in situ through slots in the cavity wall. It is evident that the radical concentration increases during the light periods, and remains constant during the dark periods.

It is obvious that the radicals are connected with the oxidation of some resin components by air oxygen and with the irradiation of the samples by light. Colophony is reported to take up oxygen easily, especially when the surface of the specimen is large as in a powdered form ${ }^{1}$. When photosensitized by dyes as eosine, the resin acids structurally related to levopimaric acid have been shown to add oxygen forming peroxide derivatives ${ }^{2}$. It is believed that the radical content in the resin products is connected in some way with such reactions. This assumption is supported by the fact that more or less crude preparations of abietic acids exhibited an ESRsignal and an analogous behaviour as colophony when melted and solidified. In

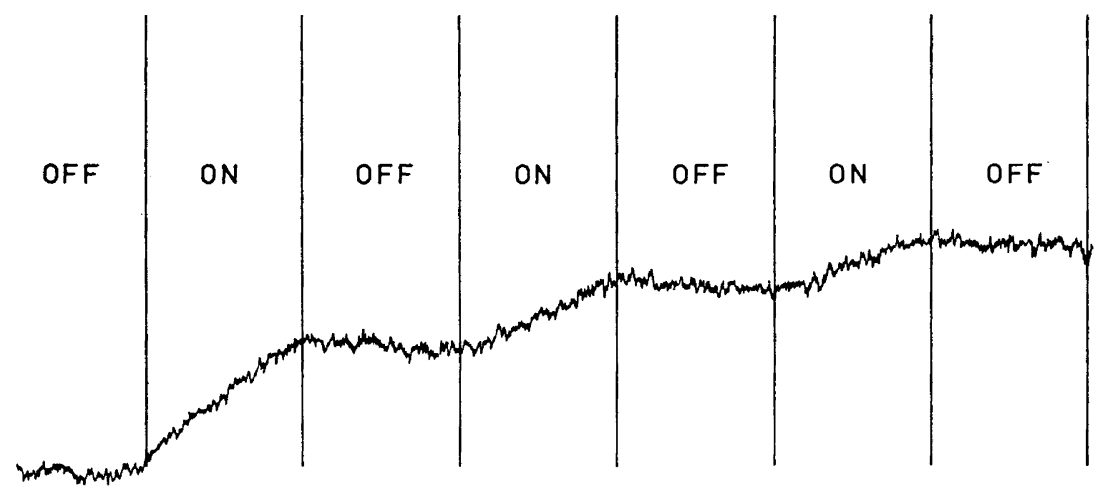

Fig. 1. Light induced free radicals in a solid sample of colophony. Successive periods of light on and off are of $5 \mathrm{~min}$ duration. Unfiltered light from a xenon lamp.

Acta Chem. Scand. 16 (1962) No. 2 


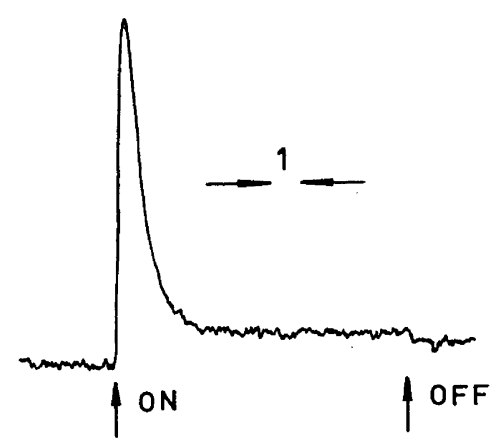

Fig. 2. Light induced free radicals in a solution of levopimaric acid $\left(10^{-2} \mathrm{M}\right)$ in ethanol containing eosine $\left(10^{-3} \mathrm{M}\right)$. Time between horizontal arrows $=1 \mathrm{~min}$. Light from a xenon lamp filtered through a $50 \mathrm{~mm}$ layer of $10 \% \mathrm{CuSO}_{4}$ solution in $\mathrm{H}_{2} \mathrm{O}$.

contrary no ESR-signal could be detected in a solid sample of dehydroabietic acid, which is reported to be resistent to air oxidation 1 .

Further evidence of a free radical mecha. nism in the reactions discussed was obtained as follows. A sample of highly purified levopimaric acid in ethanol containing eosine as a sensitizer was placed in the ESR-cavity and irradiated in situ by visible light as described above. Fig. 2 shows the relative radical concentration as a function of time. The magnetic field was kept constant after being adjusted to maximum response in a previous record. When the light was turned on, there was an immediate rise of the radical concentration, probably very much faster than the time response of the recorder (2 sec.). After having passed through a maximum the radical concentration decays almost completely in a few minutes. Degassing of the solution before the reaction does not seem to alter the shape of the response curve. It was noted that the solution was completely decolourized in the irradiated area of the sample cell. No response could be detected in an analogous experiment performed with pimaric acid.

As long as the resins are in the solid state or in a rigid solution as in colophony, the radicals formed from levopimaric and structurally related resin acids are trapped in situ. When the viscosity is lowered by heating or the substances are melted, the radicals become mobile enough to react and disappear. The lack of an ESR-signal of a solution of colophony in xylene is also consistent with this conception. The presence of radicals in a solution of colophony in alkaline ethanol might be interpreted as due to semiquinone radicals derived from transannular peroxides of the resin acids, as semiquinone radicals are known to be stabilized in an alkaline medium.

The process of formation and trapping of free radicals by light irradiation and by oxygen uptake, and their subsequent disappearence when the viscosity decreases, can probably be recycled in natural resins as long as there are substances available which are of the type here considered.

The structure of the radicals related to the resin acids is unknown at present, nor is it clear if the radicals generated by light are identical or not with those formed by air oxidation.

The ESR-spectra were obtained by use of a Varian $100 \mathrm{kc}$ spectrometer equipped with the multipurpose cavity with radiation slots. The irradiation experiments on liquid solutions were performed by use of the flat cell.

The authors are indebted to Dr. H. Bruun for samples of levopimaric and pimaric acid, to Dr. K. J. Karrman for a sample of dehydroabietic acid, and to Wilhelm och Martina Lundgrens Vetenskapsfond for financial support.

1. Sandermann, W. Naturharze, Terpentinöl, Tallöl, Berlin 1960.

2. Schuller, W. H. and Lawrence, R. V. $J$. Am. Chem. Soc. 83 (1961) 2563.

Received January 23, 1962.

\section{Correction to "A Method for Gas-} Liquid Multiplicative Distribution on a Preparative Scale" * $O \mathrm{RN}$ WAH L R OOS

\section{Laboratory of the Foundation for Chemical Research, Biochemical Institute, Helsinki, Finland}

$\mathrm{O}_{\mathrm{s}}$ p. 2055, formula (4) is incorrect and hould read

$$
\begin{aligned}
& n=\sqrt{n_{1} n_{2}}=\frac{b^{2}}{a^{*}+\left(1 / a^{*}\right)-2} \\
& \text { Received January 31, } 1962 .
\end{aligned}
$$

* Acta Chem. Scand. 15 (1961) 2053. 